\title{
MEGAGAUSS CYCLOTRON RESONANCE AND QUANTUM HALL EFFECT OF 2D ELECTRON GAS IN HgCdMnTe ${ }^{\dagger}$
}

\author{
G. Grabecki, S. Takeyama \\ Himeji Institute of Technology, Faculty of Science \\ 1479-1 Kanaji, Kamigori-cho, Akogun, Hyogo, 678-12 Japan \\ T. DIETL \\ Institute of Physics, Polish Academy of Science \\ Al. Lotników 32/46, 02-668 Warszawa, Poland \\ T. Takamasu, Y. Shimamoto and N. MiUra \\ Institute for Solid State Physics, University of Tokyo \\ Roppongi, Minato-ku, Tokyo 106, Japan
}

\begin{abstract}
Two-dimensional electron gas adjacent to a grain boundary in bicrystal of narrow-gap semiconductor $p$ - $\mathrm{Hg}_{0.79} \mathrm{Cd}_{0.19} \mathrm{Mn}_{0.02} \mathrm{Te}$ has been studied under ultra strong impulse magnetic fields (up to $140 \mathrm{~T}$ ). Both cyclotron resonance and quantum Hall effect are measured for the same samples. The values of the resonance fields point to strong nonparabolicity. A broadening of the line is interpreted in terms of an intersubband mixing that occurs for the upper Landau level. A steep increase in the linewidth in the field range 20-30 T, which coincides with a strong decrease in the Hall resistance is assigned to the field-induced metal-insulator transition in our system.
\end{abstract}

PACS numbers: 71.25.Hc, 73.50.Fq, 73.20.Dx, 73.40.Lq

In the present work, we report on a study of the electron cyclotron resonance (CR) and the quantum Hall effect (QHE) in a bicrystal of a narrow-gap semiconductor $p$ - $\mathrm{Hg}_{0.79} \mathrm{Cd}_{0.19} \mathrm{Mn}_{0.02} \mathrm{Te}$, in the magnetic fields up to $140 \mathrm{~T}$. Electrical properties of the electron inversion layers at grain boundaries (GB) in narrow-gap $\mathrm{Hg}_{1-x-y} \mathrm{Cd}_{x} \mathrm{Mn}_{y} \mathrm{Te}$ are rather well known due to the extensive transport studies [1]. The application of ultra high magnetic fields to narrow gap systems, due to a low value of the electron effective mass $m^{*}$, and hence, very large Landau level

†This work was partially supported under Hyogo Science Technology Association, contract No. 6W35 and the State Committee for Scientific Research (Republic of Poland) through grant PBZ 101. The financial support of the Japan Society for Promotion of Science for one of the authors (G.G.) is gratefully acknowledged. 
(LL) splittings allows to achieve the unique situation, for which the cyclotron energy $\hbar \omega_{c}$ is greater than both the quantum well (QW) depth $V_{0}$, and the energy gap $E_{\mathrm{g}}$.

The same bicrystal as that previously examined by magnetotransport measurements up to $20 \mathrm{~T}$ [2] is a subject of the present study. For this material, $E_{\mathrm{g}}$ was determined by optical absorption to be $180 \pm 5 \mathrm{meV}$ at $4.2 \mathrm{~K}$, while Hall effect measurements yielded 2D-electron concentration $N_{\mathrm{s}}=1.1 \times 10^{12} \mathrm{~cm}^{-2}$, and the Hall mobility of $2 \times 10^{4} \mathrm{~cm}^{2} /(\mathrm{V} \mathrm{s})$.

For the CR measurements, the megagauss magnetic fields were generated by means of a single-turn-coil (destructive) technique described in detail elsewhere [3]. Infrared transmission experiments were performed using either $\mathrm{CO}_{2}$ (energy range 117-134 meV) or CO (energy range 215-235 meV) lasers, with typical beam power $10 \mathrm{~mW} / \mathrm{mm}^{2}$. The QHE has been measured using long-pulse, nondestructive method [4].

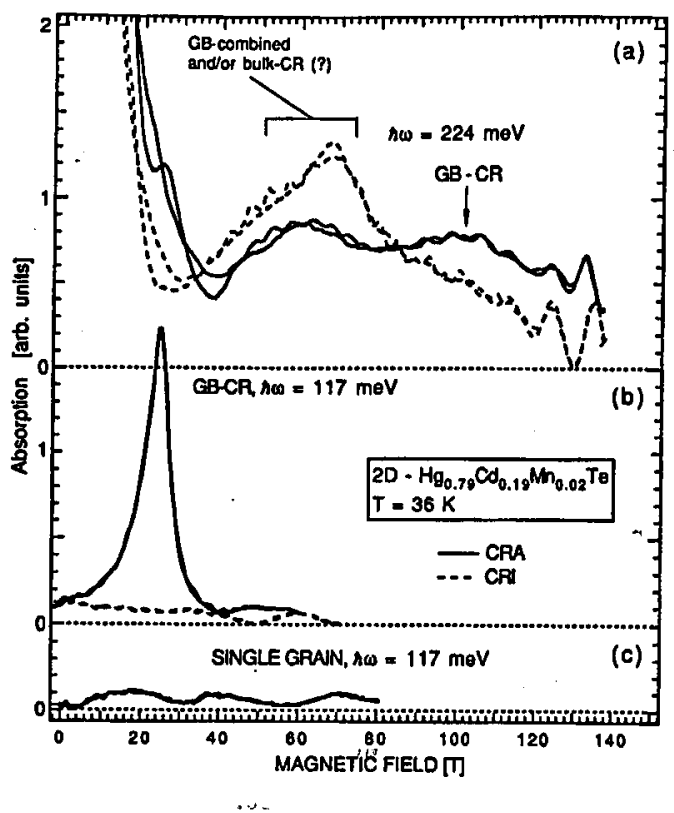

Fig. 1. CR spectra for one of the GBs taken at two different radiation energies (a) $\hbar \omega=224 \mathrm{meV}$, (b) $\hbar \omega=117 \mathrm{meV}$. Part (c) represents the result for reference single-grain sample. Solid lines represent electron active (CRA), dashed - hole active (CRI) polarization.

The low-temperature CR spectra for the single-grain sample and one of the GBs are compared in Fig. 1. Two curves indicate the magnetic field sweeps up and down respectively, and serve to illustrate the degree of the reproducibility in the data. A pronounced, single absorption line (e.g. the transmission change reaching $70 \%$ for $\hbar \omega=117 \mathrm{meV}$ ) is visible only for the bicrystalline sample in the electron active (CRA) polarization, proving that we have observed CR originating 


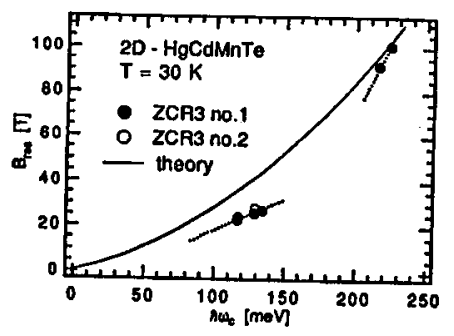

a)

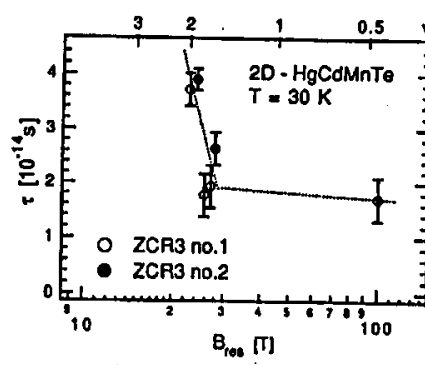

b)

Fig. 2. (a) Energy dependence of the resonance field position for two measured samples. Continuous line is theoretically calculated energy difference between the lowest LLs. (b) Electron lifetime as a function of the resonant field for the same samples. Dotted lines are guides for the eyes.

from the GB electrons. For $h \Omega=224 \mathrm{meV}$ which is greater than $E_{\mathrm{g}}$, the sample becomes transparent in the fields higher than $20 \mathrm{~T}$ due to the Landau shift of both the conduction and valence band edges in the surrounding bulk. The CR line near $100 \mathrm{~T}$ is significantly wider than that observed at lower energy. The CR measurements in tilted magnetic fields (up to the tilt angle $45^{\circ}$ ) have shown that for $\hbar \omega \approx 117 \mathrm{meV}$ the $\mathrm{CR}$ peak position remains unchanged. Such 3-dimensional-like beliavior is predicted for the high field CR in narrow-gap 2D systems, where the energy of the magnetic quantization is greater than that of electric quantization, i.e., $\hbar \omega_{\mathrm{c}} \gg V_{0}[5]$.

The energy dependence of the resonance field $B_{\text {res }}$ is presented in Fig. 2a for two independently measured samples. The estimated values of the cyclotron effective mass are equal to $0.023 \pm 0.002$ for resonance energy $\hbar \omega=117 \mathrm{meV}$ and $0.052 \pm 0.004$ for $\hbar \omega=224 \mathrm{meV}$. We have compared them with an existing theoretical model of the electron states at the GB in a narrow-gap semiconductor alloy, described in detail previously [6]. This model is based on self-consistent calculation within the 3 -band $\boldsymbol{k} \cdot \boldsymbol{p}$ approximation. The calculated energy difference between the lowest LL $\left(1^{-}-0^{-}\right)$as a function of magnetic field has been plotted in Fig. 2a. As one can see, the theory predicts somehow different shape of the nonparabolicity than that observed experimentally.

The line widths at half maximum $\Delta B$ are rather large, $\Delta B=7 \mathrm{~T}$ at $\hbar \omega=$ $117 \mathrm{meV}$, and $\Delta B=35 \mathrm{~T}$ at $\hbar \omega=224 \mathrm{meV}$. The corresponding mobility value at $B=24 \mathrm{~T}$ is equal to $2900 \mathrm{~cm}^{2} /(\mathrm{V} \mathrm{s})$, which is about seven times smaller than the value obtained from low-field transport measurements. Apart from the strong 
nonparabolicity and possible sample inhomogeneity, a strong broadening of the CR line seems to be mainly caused by a resonance character of the upper LL. In the $\mathrm{QW}$ at the GB, the theory predicts a decrease in the intersubband spacing with the subband index. Thus, for very large LL splitting, the upper LL $\left(1^{-}\right)$is degenerate with the continuum of the lowest LLs of the upper subbands.

The obtained value of the electron lifetime $\tau$ are collected in Fig. 2b. A steep decrease in $\tau$ in the magnetic field range between 20 and $30 \mathrm{~T}$ is visible for both studied samples. One of possible explanations of such behavior could be a magnetic field-induced localization of the $2 d$-electrons on donors at the GB plane. This seems possible, because at $B=30 \mathrm{~T}$, the magnetic length $l_{B}$ is $50 \AA$, which is about 2 times smaller than the average distance between the donors at the GB plane. Such a transition to the strongly localized regime has been observed by Zrenner et al. in a similar system, $\mathrm{GaAs} \delta$-doped layers, under the same conditions [7]. They have observed a strong increase in the both longitudinal $R_{x x}$ and Hall resistances $R_{x y}$ indicating a transition in an insulating state. To confirm whether this effect also occurs in our case, we have performed QHE measurements on the same GB. We have found that only $R_{x x}$ behaves in a way similar to the $\delta$-doped layers. Surprisingly, the $R_{x y}$ exhibits an opposite behavior, it abruptly decreases in the same field range.

\section{References}

[1] G. Grabecki, T. Dietl, J. Kossut, P. Sobkowicz, W. Zawadzki, Appl. Phys. Lett. 45, 1214 (1984); T. Suski, P. Wisniewski, P. Dmowski, G. Grabecki, T. Dietl, J. Appl. Phys. 65, 1203 (1989).

[2] G. Grabecki, A. Wittlin, T. Dietl, P.A.A. Teunissen, S.A.J. Wiegers, J.A.A.J. Perenboom, Semicond. Sci. Technol. 8, S95 (1993).

[3] M. Miura, T. Goto, K. Nakao, S. Takeyama, T. Sakakibara, T. Haruyama, T. Kikuchi, Physica B 155, 23 (1989).

[4] S. Takeyama, H. Ohimizu, S. Sasaki, N. Miura, Meas. Sci. Tech. (UK) 3, 662 (1992).

[5] W. Zawadzki, S. Klahn, U. Merkt, Phys. Rev. B 33, 6916 (1986); J.H. Crasemann, U. Merkt, Solid State Commun. 47, 917 (1983); J. Singleton, F. Nasir, R.J. Nicholas, Surf. Sci. 170, 409 (1986).

[6] P. Sobkowicz, G.Grabecki, P. Wisniewski, T. Suski, T. Dietl, in: Proc. 19 ICPS Warsaw (1988), Ed. W. Zawadzki, Inst. of. Phys. Polish Acad. of Sci., Warsaw 1988 , p. 611.

[7] A. Zrenner, F. Koch, J. Leotin, M. Goiran, K. Ploog, Semicond. Sci. Technol. 3, 1132 (1988). 